\title{
Strain relaxation and phonon confinement in self-assembled InAsSb/InP (001) quantum dashes: Effect of deposition thickness and composition
}

\author{
W. Lei, ${ }^{1, a)}$ H. H. Tan, ${ }^{1}$ C. Jagadish, ${ }^{1}$ Q. J. Ren, ${ }^{2}$ J. Lu, ${ }^{2}$ and Z. H. Chen ${ }^{2}$ \\ ${ }^{1}$ Department of Electronic Materials Engineering, RSPE, The Australian National University, Canberra, \\ ACT 0200, Australia \\ ${ }^{2}$ Department of Physics and National Key Laboratory for Surface Physics, Fudan University, \\ Shanghai 200433, People's Republic of China
}

(Received 10 June 2010; accepted 4 November 2010; published online 2 December 2010)

\begin{abstract}
This paper presents a study on the strain relaxation and phonon confinement effect in InAsSb/InP quantum dashes (QDashes). The phonon mode with a frequency between that of InAs-like longitudinal optical mode and that of InP transverse optical mode is determined to be originated from InAsSb QDashes. Despite the small height of the QDashes, their phonon frequency is found to be mainly determined by the strain relaxation in the dashes. With increasing InAsSb deposition thickness and Sb composition in InAsSb dashes, the phonon mode shows an upward shift of its frequency due to the increased compressive strain. (C) 2010 American Institute of Physics. [doi:10.1063/1.3522889]
\end{abstract}

In the past several years, self-assembled InAsSb nanostructures such as quantum dots (QDs), quantum dashes (QDashes), and quantum wires (QWRs), have attracted much attention due to their potential as midinfrared emitters for applications in military, telecommunications, molecular spectroscopy, biomedical surgery, environmental protection, and manufacturing industry. ${ }^{1-5}$ Compared with $\mathrm{In}(\mathrm{Ga}) \mathrm{As}$ nanostructures on GaAs or InP substrates, these InAsSb nanostructures have much smaller island sizes $(\sim 1-3 \mathrm{~nm}$ in height) due to the large lattice mismatch between InAsSb and GaAs or InP materials. ${ }^{5-8}$ The large strain and strong spatial confinement (induced by the small island height) in this material system will have a significant influence on the optical and electronic properties of these InAsSb nanostructures, and thus their device performance. Therefore, it is crucial to understand the role of strain relaxation and confinement effect in these material systems.

Despite the small scattering volume of nanostructures relative to matrix material, Raman spectroscopy has been used as an effective approach to probe the strain relaxation and phonon confinement in $\mathrm{In}(\mathrm{Ga}) \mathrm{As}$ QDs and QWRs. ${ }^{9-12}$ In this work, we present a study on the strain relaxation and phonon confinement in InAsSb/InP QDashes using Raman scattering. A phonon mode observed with a frequency between that of InAs-like longitudinal optical (LO) mode and InP transverse optical (TO) mode is determined to be originated from InAsSb QDashes. The phonon confinement and strain relaxation in InAsSb QDashes with various InAsSb deposition thickness and $\mathrm{Sb}$ composition are investigated by analyzing their phonon frequency shift.

InAsSb/InP nanostructures were grown on semiinsulating $\operatorname{InP}(001)$ substrates by a horizontal flow metalorganic chemical-vapor deposition reactor (AIX200/4) at a pressure of 180 mbar. Trimethylindium, trimethylgallium, trimethylantimony, $\mathrm{PH}_{3}$, and $\mathrm{AsH}_{3}$ were used as precursors and ultrahigh purity $\mathrm{H}_{2}$ as the carrier gas. The samples were grown using the following layer sequence: first, a $200 \mathrm{~nm}$

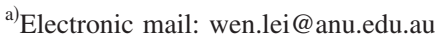

InP buffer was deposited at $650{ }^{\circ} \mathrm{C}$ followed by a $0.6 \mathrm{~nm}$ GaAs interlayer to minimize the As-P exchange. Growth was then interrupted and the temperature reduced to $520{ }^{\circ} \mathrm{C}$ to grow the InAsSb layer. The InAsSb layer was capped immediately without any growth interruption with a $100 \mathrm{~nm} \mathrm{InP}$ cap layer while the growth temperature was ramped up to $650{ }^{\circ} \mathrm{C}$. The same steps were followed to grow a top InAsSb layer for atomic force microscopy (AFM) measurements. The V/III ratio during the growth of InAsSb was set at 1.5. To study the optical phonons and strain relaxation in InAsSb nanostructures, the InAsSb deposition thickness was varied from 2 to $6 \mathrm{ML}$ and the Sb mole fraction (in the vapor phase) of InAsSb layers, quoted as nominal average $\mathrm{Sb}$ composition of InAsSb layers in the later discussion, was varied from 0.25 to 0.75 . The morphology of the top InAsSb nanostructures was characterized with AFM in tapping mode. Raman scattering measurements were performed in backscattering geometry at room temperature with a JobinYvon HR800 confocal micro-Raman system. Parallel, $z\left(x^{\prime}, x^{\prime}\right) \bar{z}$, and crossed, $z\left(x^{\prime}, y^{\prime}\right) \bar{z}$, polarization configurations $\left(x\|[100], y\|[010], z\left\|[001], x^{\prime}\right\|[110], y^{\prime} \|[1-10]\right)$ were used. The samples were excited by the $632.8 \mathrm{~nm}$ line of a $\mathrm{He}-\mathrm{Ne}$ laser to a $2 \mu \mathrm{m}$ spot on the surface with an excitation power of $4 \mathrm{~mW}$.

Figure 1(a) shows the AFM images of $\operatorname{InAs}_{0.5} \mathrm{Sb}_{0.5}$ nanostructures with 2, 4, 5, and $6 \mathrm{ML}$ of deposition. Few small and flat InAsSb QDashes are formed in the 2 ML sample. For the samples with 4,5 , and $6 \mathrm{ML}$ deposition, a high density of InAsSb QDashes is obtained. The formation of flat InAsSb QDashes could be associated with the "surfactant effect" of $\mathrm{Sb}$ atoms, which has been mentioned elsewhere. ${ }^{5}$ The "surfactant effect" may induce little higher Sb concentration in the top region of a single QDash compared with the bottom region of the QDash. ${ }^{7,8}$ However, considering the large laser spot $(2 \mu \mathrm{m})$ on the sample surface, the average $\mathrm{Sb}$ composition in the dashes is used for the discussion. The InAsSb QDashes have an average height of 0.9, 1.7, 2, and $2.4 \mathrm{~nm}$, lateral width (along the [110] direction) of 23, 25, 25 , and $25 \mathrm{~nm}$, length (along the [1-10] direction) of 70 , 


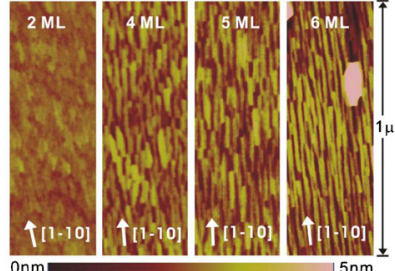

(a)

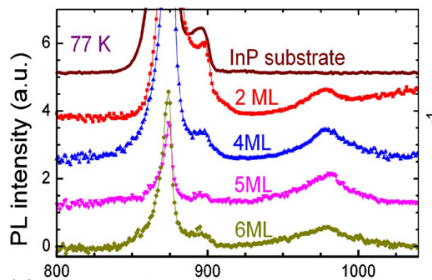

(c)

$\mathrm{PL}$ wavelength $(\mathrm{nm})$

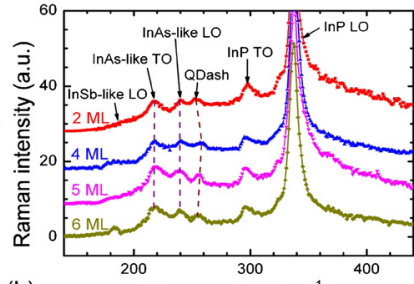

(b) Raman shift $\left(\mathrm{cm}^{-1}\right)$

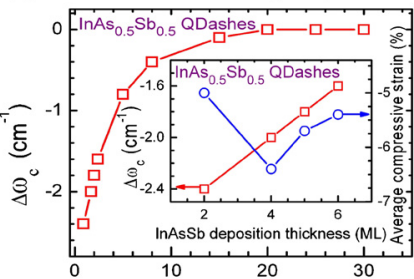

(d) InAsSb QDash height (nm)

FIG. 1. (Color online) (a) AFM images of surface $\mathrm{InAs}_{0.5} \mathrm{Sb}_{0.5}$ QDashes with various InAsSb thickness. (b) Raman spectra of $\operatorname{InAs}_{0.5} \mathrm{Sb}_{0.5}$ QDash samples with various InAsSb thicknesses measured under the $z\left(x^{\prime}, x^{\prime}+y^{\prime}\right) \bar{z}$ configuration. (c) $77 \mathrm{~K} \mathrm{PL}$ spectra of the WLs for InAs ${ }_{0.5} \mathrm{Sb}_{0.5}$ QDashes with various InAsSb thicknesses and (d) calculated confinement induced phonon frequency shift of $\operatorname{InAs} \mathrm{As}_{0.5} \mathrm{Sb}_{0.5}$ QDashes with various dash heights. The inset of (d) shows the calculated confinement induced phonon frequency shift and estimated average compressive strain in the 2, 4, 5, and $6 \mathrm{ML}$ InAsSb QDashes.

105, 150, and $300 \mathrm{~nm}$ for the 2, 4, 5, and $6 \mathrm{ML}$ samples, respectively. Figure 1(b) shows the Raman spectra of InAsSb/InP samples with various InAsSb deposition thicknesses obtained under the $z\left(x^{\prime}, x^{\prime}+y^{\prime}\right) \bar{z}$ configuration. Six Raman peaks can be observed in the frequency range of 150-400 $\mathrm{cm}^{-1}$. The phonon peaks at 296 and $338 \mathrm{~cm}^{-1}$ are from the TO and LO modes of InP matrix, while the peaks at 218 and $240 \mathrm{~cm}^{-1}$ are from the InAs-like TO and LO modes of InAsSb layer. ${ }^{9,10,12}$ Below the InAs-like TO mode, there is another weak Raman peak around $184 \mathrm{~cm}^{-1}$, which can be related to the InSb-like LO mode of InAsSb layer according to Ref. 13. Besides these phonon modes, there is another phonon peak above the InAs-like LO mode (253-258 $\left.\mathrm{cm}^{-1}\right)$. Considering the materials grown in the samples, there are three possible origins for this phonon peak: InAsSb wetting layer (WL), GaAs interlayer, and InAsSb QDashes. It is noted that its frequency varies with increasing InAsSb thickness $\left(253,258,256\right.$, and $255 \mathrm{~cm}^{-1}$ for 2, 4, 5, and $6 \mathrm{ML}$ InAsSb QDashes), while the frequencies of other phonon peaks remain constant with varying InAsSb thickness. It has been reported that in self-assembled growth of nanostructures the WL thickness remains constant once the islands start forming, where its thickness is determined by the lattice mismatch between epitaxy layer and substrate. ${ }^{11,14}$ Because of the same nominal average Sb composition in InAsSb layers, the InAsSb dashes with 2, 4, 5, and $6 \mathrm{ML}$ thickness will have WLs with the same thickness and thus the same strain and confinement effect, which is confirmed by the photoluminescence (PL) spectra of these WLs. Figure 1(c) shows the 77 K PL spectra of the WLs of InAsSb dashes with various thicknesses. The PL peaks at 874 and $895 \mathrm{~nm}$ are from the InP substrate, while the PL peak at $979 \mathrm{~nm}$ is from the InAsSb WLs. ${ }^{15}$ Clearly, the PL peak of InAsSb WLs does not shift with increasing InAsSb deposition thickness, indicating the same thickness and thus the same strain and confinement effect for the WLs. Similarly, because GaAs interlayer thickness is the same for all samples, the strain and confinement effect should also be around the same. Therefore, the phonon mode around $253-258 \mathrm{~cm}^{-1}$ can be determined to arise from the InAsSb QDashes, the frequency of which changes with increasing InAsSb deposition thickness. ${ }^{11}$ The InAs-like TO, InAs-like LO, and InSb-like LO modes, on the other hand, can be considered to originate from the InAsSb WL.

To understand the frequency shift of QDash phonon mode with increasing InAsSb deposition thickness, both strain relaxation and phonon confinement effect in InAsSb QDashes have to be taken into account. For a perfect heterostructure, both compressive strain (upward shift) and confinement effects (downward shift) play a dominant role in the observed phonon frequency shift $\left(\Delta \omega_{\exp }\right):\left(\Delta \omega_{\exp }=\Delta \omega_{\text {strain }}\right.$ $\left.+\Delta \omega_{c}\right) \cdot{ }^{12,16}$ To evaluate the phonon confinement effect in InAsSb nanostructures, the first order Raman spectrum $I(\omega)$ is calculated using the modified spatial correlation model: ${ }^{17,18} I(\omega) \cong \int\left(\left[d^{3} q|C(0, q)|^{2}\right] /\left\{[\omega-\omega(q)]^{2}+\left(\Gamma_{0} / 2\right)^{2}\right\}\right)$, where $I(\omega),|C(0, q)|^{2}, \omega(q), \Gamma_{0}, \omega$, and $q$ are the Raman intensity, Fourier coefficients, phonon dispersion curve, natural line-width, phonon frequency, and phonon wavevector, respectively. Depending on the shape and size of the nanostructures, Fourier coefficients $|C(0, q)|^{2}$ have different relationships with nanostructure size. Considering the large length $(>70 \mathrm{~nm})$ and large lateral width $(>20 \mathrm{~nm})$ of InAsSb QDashes, thin film equation ${ }^{18}\left[\left|C\left(0, q_{1}\right)\right|^{2}\right.$ $\cong e^{-q_{1}^{2} L_{1}^{2} / 16 \pi^{2}}\left|1-\operatorname{erf}\left(i q_{1} L_{1} / \sqrt{32 \pi}\right)\right|^{2}$, where $L_{1}$ is the film thickness] is utilized as the Fourier coefficient in the calculation. Figure 1(d) shows the calculated confinement induced phonon frequency shift for $\operatorname{InAs}_{0.5} \mathrm{Sb}_{0.5}$ QDashes with various heights. The downward phonon frequency shift induced by confinement effect is around $2.4,2,1.8$, and $1.6 \mathrm{~cm}^{-1}$ for the 2, 4, 5, and $6 \mathrm{ML}$ InAsSb QDashes, respectively. Considering the large phonon frequency shift $\left(>15 \mathrm{~cm}^{-1}\right)$ of InAsSb dashes relative to that of InAs LO mode in bulk materials $\left(239 \mathrm{~cm}^{-1}\right){ }^{13}$ strain relaxation in $\mathrm{InAsSb}$ dashes plays a more dominant role in determining the phonon frequency of InAsSb dashes than the height (size) of the dashes. To evaluate the strain relaxation in InAsSb dashes, a model proposed in Ref. 12 is applied to calculate the average compressive strain in the InAsSb dashes. By using the parameters listed in Refs. 12, 16, and 19, the average compressive strain is estimated to be around $5.0 \%, 6.4 \%, 5.7 \%$, and $5.4 \%$ for 2, 4, 5, and $6 \mathrm{ML}$ InAsSb dashes, respectively, which are all smaller than the maximum lattice mismatch $(6.8 \%)$ between $\mathrm{InAs}_{0.5} \mathrm{Sb}_{0.5}$ and InP. The average compressive strain in InAsSb dashes increases from $5.0 \%$ to $6.4 \%$ with increasing InAsSb deposition from 2 to $4 \mathrm{ML}$, which is usually expected for self-assembled growth. ${ }^{10,11}$ However, when the InAsSb deposition increases further from $4 \mathrm{ML}$ to 5 and 6 $\mathrm{ML}$, the average compressive strain decreases from $6.4 \%$ to $5.7 \%$ and $5.4 \%$, respectively, which may be associated with other reasons such as defects. As shown in Fig. 1(a), some large and dislocated islands are formed in the $6 \mathrm{ML}$ InAsSb sample, which can significantly relax the strain accumulated in the QDashes, ${ }^{10}$ and lead to a downward frequency shift observed. Though no dislocated islands are observed with AFM for the $5 \mathrm{ML}$ InAsSb sample, the existence of defects is still possible due to the highly accumulated strain, which needs a further investigation.

To investigate the effect of $\mathrm{Sb}$ composition in $\mathrm{InAsSb}$ dashes on the strain relaxation, the nominal average $\mathrm{Sb}$ composition in InAsSb dashes is varied from $25 \%$ to $75 \%$. Figure 


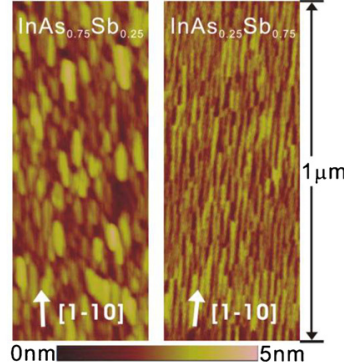

(a)

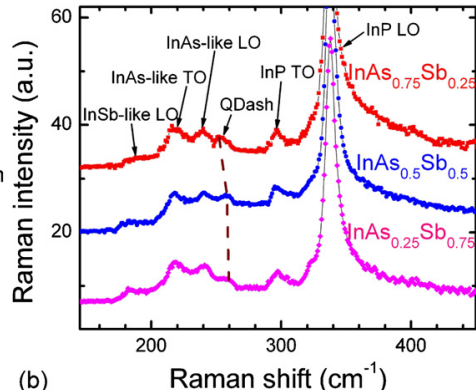

FIG. 2. (Color online) (a) AFM images of $4 \mathrm{ML}$ surface $\operatorname{In} \mathrm{As}_{0.75} \mathrm{Sb}_{0.25}$ and InAs ${ }_{0.25} \mathrm{Sb}_{0.75}$ QDashes. (b) Raman spectra of $4 \mathrm{ML}$ InAs $\mathrm{s}_{0.75} \mathrm{Sb}_{0.25}$, InAs ${ }_{0.5} \mathrm{Sb}_{0.5}$, and $\operatorname{InAs}_{0.25} \mathrm{Sb}_{0.75}$ QDash samples measured under the $z\left(x^{\prime}, x^{\prime}+y^{\prime}\right) \bar{z}$ configuration.

2(a) shows the AFM images of $\operatorname{InAs}_{0.75} \mathrm{Sb}_{0.25}$ and InAs $s_{0.25} \mathrm{Sb}_{0.75}$ QDashes. The InAs $\mathrm{As}_{0.75} \mathrm{Sb}_{0.25}$ QDashes show an average height, width, and length of $1.9,30$, and $92 \mathrm{~nm}$, while the $\operatorname{InAs}_{0.25} \mathrm{Sb}_{0.75}$ QDashes an average height, width, and length of 1.3, 18, and $160 \mathrm{~nm}$. Figure 2(b) shows the Raman spectra of InAsSb samples with various nominal average $\mathrm{Sb}$ compositions obtained under $z\left(x^{\prime}, x^{\prime}+y^{\prime}\right) \bar{z}$ configuration. Besides InP LO and TO modes, four other modes are also observed in Fig. 2(b). Because of the different nominal average $\mathrm{Sb}$ compositions in InAsSb layers, the WLs will present different strains and thicknesses (thus confinement effects), which are demonstrated by the slight shift of the PL peak of WLs with increasing Sb composition (PL results not shown here). However, based on the dash sizes measured and the nominal average $\mathrm{Sb}$ compositions, the frequency shifts of QDash phonon caused by the change of confinement/strain effect, relative to the phonon frequency of $\mathrm{InAs}_{0.5} \mathrm{Sb}_{0.5}$ QDashes, are estimated to be $\sim 0.2 /-5.2$ and $-0.2 / 4 \mathrm{~cm}^{-1}$ for $\operatorname{InAs}_{0.75} \mathrm{Sb}_{0.25}$ and $\operatorname{InAs}_{0.25} \mathrm{Sb}_{0.75}$ QDashes according to the modified spatial correlation model and strain model discussed. ${ }^{12,16-19}$ Therefore, the phonon modes observed at 253 and $260 \mathrm{~cm}^{-1}$ in Fig. 2(b) can be determined to arise from InAs ${ }_{0.75} \mathrm{Sb}_{0.25}$ and $\operatorname{InAs}_{0.25} \mathrm{Sb}_{0.75}$ dashes. No obvious frequency shifts are observed for the InAs-like LO, InAs-like TO, and InSb-like LO phonon modes of InAsSb WLs with increasing $\mathrm{Sb}$ composition; the reason of which is not clear and present a subject for future study. Similarly, theoretical calculations have also been done to evaluate the phonon confinement effect and strain relaxation in InAsSb QDashes with various nominal average $\mathrm{Sb}$ compositions; the results of which are shown in Fig. 3(a). It is observed that for InAsSb QDashes with various $\mathrm{Sb}$ compositions, the strain relaxation also plays a determinant role in determining the phonon frequency (despite their small island heights the confinement
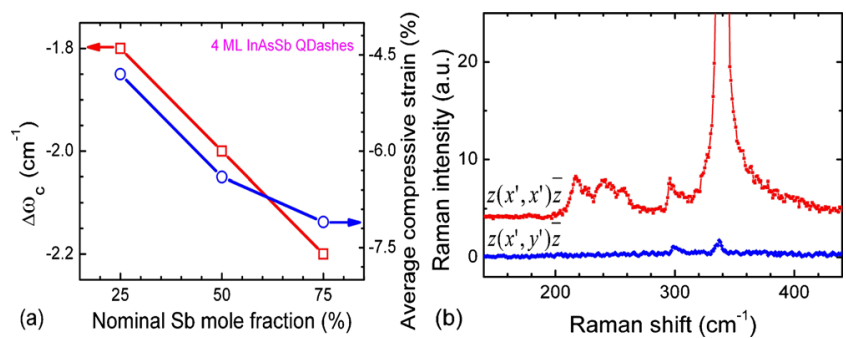

FIG. 3. (Color online) (a) Calculated confinement induced phonon frequency shift and estimated average compressive strain in $4 \mathrm{ML}$ InAs ${ }_{0.75} \mathrm{Sb}_{0.25}$, InAs ${ }_{0.5} \mathrm{Sb}_{0.5}$, and $\mathrm{InAs}_{0.25} \mathrm{Sb}_{0.75}$ QDashes. (b) polarized Raman spectra of $4 \mathrm{ML}$ InAs ${ }_{0.5} \mathrm{Sb}_{0.5}$ QDash sample. effect only induces a phonon frequency shift around $2 \mathrm{~cm}^{-1}$ relative to that of InAs LO mode in bulk materials). The average compressive strain can be estimated to be $4.8 \%$, $6.4 \%$, and $7.1 \%$ for $\operatorname{InAs}_{0.75} \mathrm{Sb}_{0.25}$, $\operatorname{InAs}_{0.5} \mathrm{Sb}_{0.5}$, and $\operatorname{InAs}_{0.25} \mathrm{Sb}_{0.75}$ QDashes, respectively. Clearly, the average compressive strain in InAsSb QDashes increases with increasing nominal average $\mathrm{Sb}$ composition, which is mainly caused by the increased lattice mismatch between InAsSb and $\mathrm{InP}$ due to large size of $\mathrm{Sb}$ atoms relative to As atoms despite that the change of dash size also has little influence.

Figure 3(b) shows the polarized Raman spectra of the 4 ML InAs ${ }_{0.5} \mathrm{Sb}_{0.5}$ QDashes. The InAsSb QDash phonon mode shows obvious polarization dependence as observed in the $z\left(x^{\prime}, x^{\prime}\right) \bar{z}$ configuration but not in the $z\left(x^{\prime}, y^{\prime}\right) \bar{z}$ configuration. This demonstrates that the QDash phonons follow the selection rules of the LO phonons in the bulk zinc-blende semiconductors. ${ }^{10,11}$

In summary, we have studied the strain relaxation and phonon confinement effect in InAsSb/InP QDashes by Raman scattering. The phonon frequency of InAsSb QDashes is found to be mainly determined by the strain relaxation rather than the small height of dashes. The increase of InAsSb deposition thickness and $\mathrm{Sb}$ composition of InAsSb alloys usually leads to an increase of compressive strain in the dashes and thus an upward shift of its phonon frequency. Additionally, the InAsSb QDash phonons are observed to follow the selection rules of LO phonons in bulk zinc-blende semiconductors.

Financial support from Australian Research Council (DP0774366) is gratefully acknowledged. Facilities used in this work are supported by the ANFF.

${ }^{1}$ C. Cornet, F. Doré, A. Ballestar, J. Even, N. Bertru, A. Le Corre, and S. Loualiche, J. Appl. Phys. 98, 126105 (2005).

${ }^{2}$ Y. Qiu and D. Uhl, Appl. Phys. Lett. 84, 1510 (2004)

${ }^{3}$ F. Doré, C. Cornet, P. Caroff, A. Ballestar, J. Even, N. Bertru, O. Dehaese, I. Alghoraibi, H. Folliot, R. Piron, A. Le Corre, and S. Loualiche, Phys. Status Solidi C 3, 3920 (2006).

${ }^{4}$ F. Doré, C. Cornet, A. Schliwa, A. Ballestar, J. Even, N. Bertru, O. Dehaese, I. Alghoraibi, H. Folliot, R. Piron, A. Le Corre, and S. Loualiche, Phys. Status Solidi C 3, 524 (2006).

${ }^{5}$ W. Lei, H. H. Tan, and C. Jagadish, Appl. Phys. Lett. 95, 013108 (2009).

${ }^{6}$ W. Lei, H. H. Tan, and C. Jagadish, Appl. Phys. Lett. 95, 143124 (2009).

${ }^{7}$ K. Kawaguchi, M. Ekawa, T. Akiyama, H. Kuwatsuka, and M. Sugawara J. Cryst. Growth 291, 154 (2006).

${ }^{8}$ K. Kawaguchi, M. Ekawa, T. Akiyama, H. Kuwatsuka, and M. Sugawara, J. Cryst. Growth 298, 558 (2007).

${ }^{9}$ S. Marcinkevičius, Y. Qiu, R. Leon, J. Ibáñez, R. Cuscó, and L. Artús, Appl. Phys. Lett. 86, 181110 (2005).

${ }^{10}$ W. Lei, Y. H. Chen, B. Xu, X. L. Ye, Y. P. Zeng, and Z. G. Wang, Nanotechnology 16, 1974 (2005).

${ }^{11}$ L. Artús, R. Cuscó, S. Hernández, A. Patanè, A. Polimeni, M. Henini, and L. Eaves, Appl. Phys. Lett. 77, 3556 (2000).

${ }^{12}$ I. Rasnik, M. J. S. P. Brasil, F. Cerdeira, C. A. C. Mendonca, and M. A Cotta, J. Appl. Phys. 87, 1165 (2000).

${ }^{13}$ Y. B. Li, S. S. Dosanjh, I. T. Ferguson, A. G. Norman, A. G. de Olivera, R. A. Stradling, and R. Zallen, Semicond. Sci. Technol. 7, 567 (1992).

${ }^{14}$ M. Tabuchi, S. Noda, and A. Sasaki, J. Cryst. Growth 115, 169 (1991).

${ }^{15}$ L. Landin, H. Pettersson, M. Kleverman, M. Borgström, X. Zhang, W. Seifert, and L. Samuelson, J. Appl. Phys. 95, 8007 (2004).

${ }^{16}$ B. Jusserand and M. Cardona, in Light Scattering in Solids V: Topics in Applied Physics, edited by M. Cardona and G. Güntherodt (Springer, Heidelburg, 1989), Vol. 66, pp. 124-128.

${ }^{17}$ H. Richter, Z. P. Wang, and L. Ley, Solid State Commun. 39, 625 (1981)

${ }^{18}$ I. H. Campbell and P. M. Fauchet, Solid State Commun. 58, 739 (1986)

${ }^{19}$ R. Carles, M. Saint-Cricq, J. B. Renucci, M. A. Renucci, and A. Zwick, Phys. Rev. B 22, 4804 (1980). 